\title{
Special Issue on Socio-Cognitive and Affective Computing
}

\author{
Antonio Fernández-Caballero ${ }^{1,2, *(1)}$, Pascual González ${ }^{1,2}$, María T. López ${ }^{1}$ \\ and Elena Navarro 1,2 (D) \\ 1 Departamento de Sistemas Informáticos, Universidad de Castilla-La Mancha, 02071 Albacete, Spain; \\ Pascual.Gonzalez@uclm.es (P.G.); Maria.LBonal@uclm.es (M.T.L.); Elena.Navarro@uclm.es (E.N.) \\ 2 Centro de Investigación Biomédica en Red de Salud Mental (CIBERSAM), 28029 Madrid, Spain \\ * Correspondence: Antonio.Fdez@uclm.es
}

Received: 2 August 2018; Accepted: 14 August 2018; Published: 15 August 2018

Abstract: Social cognition focuses on how people process, store, and apply information about other people and social situations. [...]

\section{Introduction}

Social cognition focuses on how people process, store, and apply information about other people and social situations. It focuses on the role that cognitive processes play in our social interactions [1]. On the other hand, the term cognitive computing is generally used to refer to new hardware and/or software that mimics the functioning of the human brain and helps to improve human decision-making [2]. In this sense, it is a type of computing with the goal of discovering more accurate models of how the human brain/mind senses, reasons, and responds to stimuli.

Thus, Socio-Cognitive Computing should be understood as a set of theoretical interdisciplinary frameworks, methodologies, methods and hardware/software tools to model how the human brain mediates social interactions. In addition, Affective Computing is the study and development of systems and devices that can recognize, interpret, process, and simulate human affects, a fundamental aspect of socio-cognitive neuroscience [3]. It is an interdisciplinary field spanning computer science, electrical engineering, psychology, and cognitive science.

Moreover, Physiological Computing is a category of technology in which electrophysiological data recorded directly from human activity are used to interface with a computing device [4]. This technology becomes even more relevant when computing can be integrated pervasively in everyday life environments [5]. Thus, Socio-Cognitive and Affective Computing systems should be able to adapt their behavior according to the Physiological Computing paradigm.

This Special Issue on Socio-Cognitive and Affective Computing aimed at integrating these various albeit complementary fields. Proposals from researchers who use signals from the brain and/or body to infer people's intentions and psychological state in smart computing systems were welcome. Designing this kind of system requires combining knowledge and methods of ubiquitous and pervasive computing, as well as physiological data measurement and processing, with those of socio-cognitive and affective computing. Papers with a special focus on multidisciplinary approaches and multimodality were especially welcome.

\section{The Papers}

A total of 41 papers were submitted to the Special Issue on Socio-Cognitive and Affective Computing. After peer review, 13 of them were accepted and published.

The first article addresses the issue of mapping between the models of affect representation to enhance both the accuracy and reliability of emotion recognition [6]. The paper proposes a procedure 
to elaborate new mappings, recommends a set of metrics for evaluation of the mapping accuracy, and delivers new mapping matrices for estimating the dimensions of a Pleasure-Arousal-Dominance model from Ekman's six basic emotions. The results are based on an analysis using three datasets that were constructed based on affect-annotated lexicons. The procedure, as well as the proposed metrics, might be used, not only in evaluation of the mappings between representation models, but also in comparison of emotion recognition and annotation results.

Two additional papers are centered on the user experience and frustration. The first focuses on the learning process of users as an essential cue in the construction of new knowledge when pursuing improvements in user experience [7]. In this paper, the interruption factor is considered in relation to interaction quality due to human-computer interaction (HCI) being seen to affect the learning process. The results obtained from 500 users in an interactive museum in Tijuana, Mexico are presented as a case study. The authors conclude that managing interruptions can enhance the $\mathrm{HCI}$, producing a positive learning process that influences user experience. The second paper investigates how to mitigate user frustration and support student learning through changes in the style in which a computer tutor interacts with a learner [8]. The study examines an approach to mitigate frustration using different etiquette strategies to change the amount of imposition feedback placed on the learner. Changing etiquette strategies from one math problem to the next led to changes in motivation, confidence satisfaction, and performance. The most effective etiquette strategies changed depending on whether the user was frustrated or not.

Next, a series of four papers introduces several devices that enable capturing physiological information from the user. Firstly, an analysis of the effects of the display type (2D vs. 3D) and viewing distance on visual fatigue during a 60-min viewing session based on electroencephalogram relative beta power, and alpha/beta power ratio is provided [9]. In this study, 20 male participants watch four videos. When the viewing distance is increased the visual fatigue is decreased in the case of the 3D display, whereas the fatigue is increased in the case of the 2D display. Moreover, there is approximately the same level of visual fatigue when watching videos in 2D or 3D from a long viewing distance. Another paper introduces the design of a new wearable photo plethysmography sensor and its assessment for mental distress estimation [10]. Temporal, morphological, and frequency markers are computed using time intervals between adjacent normal cardiac cycles to characterize pulse rate variability. The results show that temporal features present a single discriminant power between emotional states of calm and stress. Moreover, a discriminant tree-based model is used to assess the possible underlying relationship among parameters. A third paper describes a study carried out on a group of 35 participants to determine which methods and tools to recognize the emotions of computer users can be used during programming [11]. During the study, data from multiple sensors that are commonly used in methods of emotional recognition are collected. The participants were extensively questioned about the sensors' invasiveness during programming. Moreover, three methods for inducing negative emotions are proposed, and their effectiveness is verified. Lastly, a model that promotes the well-being of the elderly in their homes is presented [12]. The key is that there is no device that provides a more natural interaction than a human body; every one of us sends and receives useful information, which sometimes gets lost. Trends show that the future will be filled with pervasive IoT devices, present in most aspects of human life. We will focus on those aspects that are most important for human well-being and the devices, technologies, and interactions that may be used to collect data directly from users and measure their physiological and emotional responses.

The following paper aims at uncovering hidden communities in social networks by using a parallel programming framework like MapReduce with the objective of improving the efficiency of algorithms [13]. In this paper, nodes are mapped into communities based on the random walk in the network. The proposed approach is compared with some standard existing community detection algorithms for both synthetic and real-world datasets to examine its performance, and it is observed that the proposed algorithm is more efficient than the existing ones. 
Finally, five papers have focused their research on machine learning techniques. In the first place, a paper proposes Spark based Single Graph Mining, a Spark-based parallel frequent subgraph mining algorithm in a single large graph [14]. Extensive experiments with four different real-world datasets demonstrate that the proposed algorithm outperforms the existing Graph Mining algorithm by an order of magnitude for all datasets and can work with a lower support threshold. Then, a deep learning architecture, based on long short-term memory networks that model the inter-activity behavior in urban population, is presented [15]. The paper is related to the City4Age H2020 project that is working on the early detection of the risks related to mild cognitive impairment and frailty and on providing meaningful interventions that prevent these risks. The architecture offers a probabilistic model that allows us to predict the user's next actions and to identify anomalous user behaviors. The third article proposes a personalized path decision algorithm that is based on user habits for path planning of self-driving travel [16]. Results show that the algorithm can meet the personalized requirements of the user path selection in the path decision. Fourth, a novel approach to enhance the Feature Engineering and Selection (eFES) optimization process in machine learning is introduced [17]. eFES is built using a unique scheme to regulate error bounds and parallelize the addition and removal of a feature during training. eFES also invents local gain and global gain functions using 3D visualizing techniques to assist the feature grouping function. Results show the promising state of eFES as compared to the traditional feature selection process. Lastly, an inductive transfer learning-based framework (Uncertainty Flow) is put forward to allow knowledge transfer from a single-labeled emotion recognition task to a multi-label affective recognition task to lower the single-label dependency on affective facial analysis [18]. The authors demonstrate that Uncertainty Flow in multi-label facial expression analysis exhibits superiority to conventional multi-label learning algorithms and multi-label compatible neural networks.

Acknowledgments: This issue would not be possible without the contributions of the authors who submitted their valuable papers. We would like to thank all reviewers and the editorial team of Applied Sciences for their great work. Lastly, we acknowledge the financial support from the Spanish Ministerio de Ciencia, Innovación y Universidades, Agencia Estatal de Investigación (AEI)/European Regional Development Fund (FEDER, EU) under DPI2016-80894-R and TIN2015-72931-EXP grants, as well as from Centro de Investigación Biomédica en Red de Salud Mental (CIBERSAM) of the Instituto de Salud Carlos III.

Conflicts of Interest: The authors declare no conflict of interest.

\section{References}

1. Frith, C.D. Social cognition. Philos. Trans. R. Soc. Lond. B Biol. Sci. 2008, 363, 2033-2039. [CrossRef] [PubMed]

2. Modha, D.S.; Ananthanarayanan, R.; Esser, S.K.; Ndirango, A.; Sherbondy, A.; Singh, R. Cognitive computing. Commun. ACM 2011, 54, 62-71. [CrossRef]

3. Picard, R.W. Affective Computing; The MIT Press: Cambridge, MA, USA, 2000.

4. Fairclough, S.; Gilleade, K. Advances in Physiological Computing; Springer: Dordrecht, The Netherlands, 2014.

5. Fernández-Caballero, A.; González, P.; Navarro, E.; Cook, D.J. Pervasive computing for gerontechnology. Pervasive Mob. Comput. 2017, 34, 1-2. [CrossRef]

6. Landowska, A. Towards new mappings between emotion representation models. Appl. Sci. 2018, 8, 274. [CrossRef]

7. Rosales, R.; Castañón-Puga, M.; Lara-Rosano, F.; Evans, R.D.; Osuna-Millan, N.; Flores-Ortiz, M.V. Modelling the interruption on HCI using BDI agents with the fuzzy perceptions approach: An interactive museum case study in Mexico. Appl. Sci. 2017, 7, 832. [CrossRef]

8. Yang, E.; Dorneich, M.C. Evaluating human-automation etiquette strategies to mitigate user frustration and improve learning in affect-aware tutoring. Appl. Sci. 2018, 8, 895. [CrossRef]

9. Ramadan, M.Z.; Alhaag, M.H.; Abidi, M.H. Effects of viewing displays from different distances on human visual system. Appl. Sci. 2017, 7, 1153. [CrossRef]

10. Zangróniz, R.; Martínez-Rodrigo, A.; López, M.T.; Pastor, J.M.; Fernández-Caballero, A. Estimation of mental distress from photoplethysmography. Appl. Sci. 2018, 8, 69. [CrossRef] 
11. Wrobel, M.R. Applicability of emotion recognition and induction methods to study the behavior of programmers. Appl. Sci. 2018, 8, 323. [CrossRef]

12. Rodrigues, N.; Pereira, A. A user-centred well-being home for the elderly. Appl. Sci. 2018, 8, 850. [CrossRef]

13. Behera, R.K.; Rath, S.K.; Misra, S.; Damasevicius, R.; Maskeliunas, R. Large scale community detection using a small world model. Appl. Sci. 2017, 7, 1173. [CrossRef]

14. Qiao, F.; Zhang, X.; Li, O.; Ding, Z.; Jia, S.; Wang, H. A parallel approach for frequent subgraph mining in a single large graph using Spark. Appl. Sci. 2018, 8, 230. [CrossRef]

15. Almeida, A.; Azkune, G. Predicting human behaviour with recurrent neural networks. Appl. Sci. 2018, 8, 305. [CrossRef]

16. Chen, P.; Zhang, X.; Chen, X.; Liu, M. Path planning strategy for vehicle navigation based on user habits. Appl. Sci. 2018, 8, 407. [CrossRef]

17. Uddin, M.F.; Lee, J.; Rizvi, S.; Hamada, S. Proposing enhanced feature engineering and a selection model for machine learning processes. Appl. Sci. 2018, 8, 646. [CrossRef]

18. Bai, W.; Quan, C.; Luo, Z. Uncertainty flow facilitates zero-shot multi-label learning in affective facial analysis. Appl. Sci. 2018, 8, 300. [CrossRef]

(C) 2018 by the authors. Licensee MDPI, Basel, Switzerland. This article is an open access article distributed under the terms and conditions of the Creative Commons Attribution (CC BY) license (http:/ / creativecommons.org/licenses/by/4.0/). 